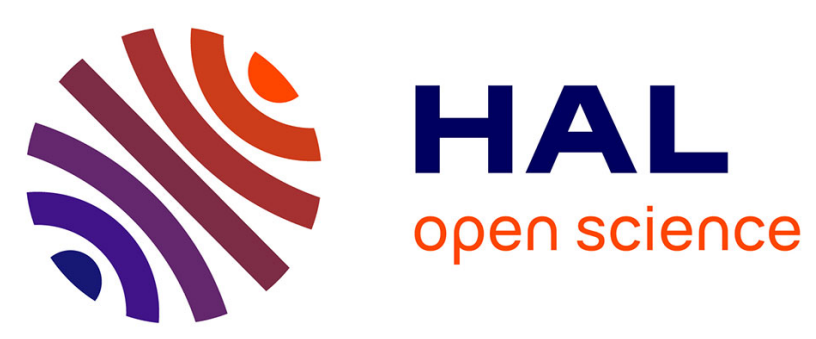

\title{
Arbitrage and asset market equilibrium in infinite dimensional economies with short-selling and risk-averse expected utilities
}

\author{
Thai Ha-Huy, Cuong Le Van, Nguyen Manh Hung
}

\section{- To cite this version:}

Thai Ha-Huy, Cuong Le Van, Nguyen Manh Hung. Arbitrage and asset market equilibrium in infinite dimensional economies with short-selling and risk-averse expected utilities. 2016. halshs-01390954

\section{HAL Id: halshs-01390954 \\ https://shs.hal.science/halshs-01390954}

Submitted on 2 Nov 2016

HAL is a multi-disciplinary open access archive for the deposit and dissemination of scientific research documents, whether they are published or not. The documents may come from teaching and research institutions in France or abroad, or from public or private research centers.
L'archive ouverte pluridisciplinaire HAL, est destinée au dépôt et à la diffusion de documents scientifiques de niveau recherche, publiés ou non, émanant des établissements d'enseignement et de recherche français ou étrangers, des laboratoires publics ou privés. 


\section{Documents de Travail du

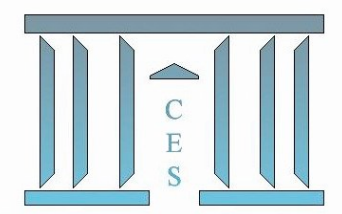

Arbitrage and asset market equilibrium in infinite dimensional economies with short-selling and risk-averse expected utilities

Thai HA-HuY, Cuong LE VAN, Nguyen MANH-Hung

2016.62

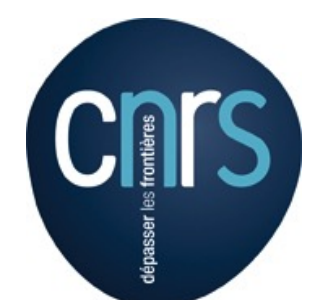




\title{
Arbitrage and asset market equilibrium in infinite dimensional economies with short-selling and risk-averse expected utilities *
}

\author{
Thai Ha-Huy ${ }^{\dagger}$ Cuong Le Van ${ }^{\ddagger}$ \\ and Manh-Hung Nguyen $\S$
}

October 17, 2015

\begin{abstract}
We consider a model with an infinite number of states of nature, von Neumann - Morgenstern utilities, where agents have different probability beliefs and where short sells are allowed. We show that no-arbitrage conditions, defined for finite dimensional asset markets models, are not sufficient to ensure existence of equilibrium in presence of an infinite number of states of nature. However, if the individually rational utility set $\mathcal{U}$ is compact, we obtain an equilibrium. We give conditions which imply the compactness of $\mathcal{U}$. We give examples of non-existence of equilibrium when these conditions do not hold.

Keywords: asset market equilibrium, individually rational attainable allocations, individually rational utility set, no-arbitrage prices, no-arbitrage condition.
\end{abstract}

JEL Classification: C62, D50, D81, D84, G1.

\footnotetext{
*The authors would like to thank Rose-Anne Dana for her thoughtful remarks and criticisms.

${ }^{\dagger} \mathrm{EPEE}$, University of Evry-Val-D’Essonne. E-mail: thai.hahuy@univ-evry.fr

${ }^{\ddagger}$ IPAG Business School, CNRS, Paris School of Economics, VCREME. E-mail: Cuong.LeVan@univ-paris1.fr

${ }^{\S}$ Toulouse School of Economics, INRA, VCREME. E-mail: mhnguyen@toulouse.inra.fr
} 


\section{Introduction}

In finite dimensional markets with short-selling, conditions on agents' utilities insuring the existence of equilibria (or equivalent to the existence of equilibria) are well understood. In particular they can be interpreted as no-arbitrage conditions. In an uncertainty setting, where agents have different beliefs and different risk aversions, as originally shown by Hart (1974), the no-arbitrage condition may be interpreted as compatibility of agent's risk adjusted beliefs. There is a huge literature on sufficient and necessary conditions for the existence of equilibria in the line of Hart's model, where the market is complete. In finite dimension, one can refer, for instance to Page (1987), Werner (1987), Nielsen (1989), Page and Wooders (1995, 1996), Allouch (1999), Allouch et alii (2000), Won and Yannelis (2008).

When the number of assets is infinite, and the market is complete, the noarbitrage condition used for finite dimension do not imply existence of equilibrium. The standard assumption is to assume that the individually rational utility set is compact ( see e.g. Cheng (1991), Brown and Werner (1995), Dana and Le Van (1996), Dana et al (1997), Dana and Le Van (2000), Le Van and Truong Xuan (2001)). ${ }^{1}$

In this paper, we consider a model with an infinite number of states of nature, a finite number of agents and Von Neumann - Morgenstern utilities with different expectations.

More precisely, we consider a model where the utility of agent $i$ is

$$
U^{i}\left(x^{i}\right)=\sum_{s=1}^{\infty} \pi_{s}^{i} u^{i}\left(x_{s}^{i}\right)
$$

where $\pi^{i}$ is her belief and $x^{i}$ is her consumption. The commodity space is $l^{p}(\pi)$ with $p \in\{1,2, \ldots,+\infty\}{ }^{2}$

\footnotetext{
${ }^{1}$ Chichilnisky and Heal (1993) give a condition which implies the boundedness of the individually rational feasible set in $L^{2}$. Since the feasible set is closed, it is therefore weakly compact in $L^{2}$.

${ }^{2}$ We use the model proposed by Hart. Investors are interested only in their wealth in the second period. We suppose that the market is complete with an asset system $\left(r^{1}, r^{2}, \ldots, r^{k}, \ldots\right)$. For each portfolio $z=\left(z^{1}, z^{2}, \ldots, z^{k}, \ldots\right)$, the wealth or investor if state $s$ occurs is

$$
w_{s}=\sum_{k} z^{k} r_{s}^{k}
$$

Her expected utility is:

$$
V\left(w^{1}, w^{2}, \ldots, w^{s}, \ldots\right)=\sum_{s} \pi_{s} u\left(w_{s}\right) .
$$

Since the market is complete, the choice of portfolio is equivalent to the choice of wealth. As in Hart's pioneer paper, we consider the expected utility function on wealth.
} 
When the number of states is infinite, the no-arbitrage condition ( $\grave{a} l a$ Werner or à la Dana-Le Van) only ensures the boundedness of the individually rational utility set. We give examples where this condition is satisfied and no equilibrium exists. The strategy is therefore to give assumptions which imply the compactness of the individually rational utility set and hence existence of equilibrium. Our conditions might be considered as among the weakest since we give also examples of non existence of equilibrium when these conditions do not hold.

The paper is organized as follows. In Section 2, we set up the model and define the equilibrium. In section 3 , we introduce no-arbitrage conditions and give conditions for the existence of equilibrium. Proofs are put in Section Appendix, Section 4. We mention that our methods of proofs are inspired by the ones in Le Van and Truong Xuan (2001). However, their model rules out the risk-neutral agents. That is not the case in our model.

\section{The model}

There are $m$ agents indexed by $i=1, \ldots, m$. The belief of agent $i$ in state $s$ is $\pi_{s}^{i} \geq 0$, and $\sum_{s=1}^{\infty} \pi_{s}^{i}=1$. Let us denote by $\pi$ the mean probability $\frac{1}{m} \sum_{i} \pi^{i}$. We first assume:

A0: $\pi^{i}$ is equivalent to $\pi^{j}$ for any $i, j$ i.e. there exists a number $h>0$ such that $h \leq \frac{\pi_{s}^{i}}{\pi_{s}^{j}} \leq \frac{1}{h}$ for all $i, j, s .^{3}$

Under A0, without loss of generality, one can assume that $\pi_{s}^{i}>0$ for any $i$, any $s$. In this paper, we always suppose that the condition $\mathbf{A 0}$ is satisfied and $\pi_{s}^{i}>0$ for any $i$, any $s$.

The consumption set of agent $i$ is $X^{i}=l^{p}(\pi)$ with $p \in\{1,2, \ldots,+\infty\}$ and agent $i$ has an endowment $e^{i} \in l^{p}(\pi)$. We assume that for each agent $i$ there exists a concave, strictly increasing, differentiable ${ }^{4}$ function $u^{i}: \mathbb{R} \rightarrow \mathbb{R}$, such that, for any $i$, the function

$$
U^{i}\left(x^{i}\right)=\sum_{s=1}^{\infty} \pi_{s}^{i} u^{i}\left(x_{s}^{i}\right)
$$

is real-valued for any $x^{i} \in X^{i}$.

Agent $i$ has $l^{p}(\pi)$ as consumption set, $e^{i}$ as initial endowment and $U^{i}$ as utility function, with $i=1, \ldots, m$.

Definition 1 An equilibrium is a list $\left.\left(\left(x^{* i}\right)_{i=1, \ldots, m}, p^{*}\right)\right)$ such that $x^{* i} \in X^{i}$ for every $i$ and $p^{*} \in l_{+}^{q}(\pi) \backslash\{0\}$ and

\footnotetext{
${ }^{3}$ With A0, the consumptions set of the agents belong to same topological space $l^{p}(\pi)$. We observe that when all agents have the same belief as in Cheng (1991), then A0 is satisfied.

${ }^{4}$ For simplicity, we assume differentiability. The results still hold by using super-gradients. But the proofs will become tedious.
} 
(i) For any $i, U^{i}(x)>U^{i}\left(x^{* i}\right) \Rightarrow \sum_{s=1}^{\infty} p_{s}^{*} x_{s}>\sum_{s=1}^{\infty} p_{s}^{*} e_{s}^{i}$,

(ii) $\sum_{i=1}^{m} x^{* i}=\sum_{i=1}^{m} e^{i}$.

Definition 2 A quasi-equilibrium is a list $\left.\left(\left(x^{* i}\right)_{i=1, \ldots, m}, p^{*}\right)\right)$ such that $x^{i *} \in X^{i}$ for every $i$ and $p^{*} \in l_{+}^{q}(\pi) \backslash\{0\}$ and

(i) For any $i, U^{i}(x)>U^{i}\left(x^{* i}\right) \Rightarrow \sum_{s=1}^{\infty} p_{s}^{*} x_{s} \geq \sum_{s=1}^{\infty} p_{s}^{*} e_{s}^{i}$,

(ii) $\sum_{i=1}^{m} x^{* i}=\sum_{i=1}^{m} e^{i}$.

In this paper, since the consumption set is the whole space, and the utility functions $U^{i}$ are continuous, any quasi-equilibrium is an equilibrium (see e.g. Geistdoerfer-Florenzano (1982), Proposition 3).

Define

$$
\begin{aligned}
& a^{i}=\inf _{x} u^{i \prime}(x)=u^{i \prime}(+\infty), \\
& b^{i}=\sup _{x} u^{i \prime}(x)=u^{i \prime}(-\infty) .
\end{aligned}
$$

Let $I_{1}$ be the set of indices $i$ such that $a^{i}<b^{i}$, and $I_{2}$ be the set of indices such that $a^{i}=b^{i}$. $I_{1}$ is the set of risk averse agents, $I_{2}$ is the set of risk neutral ones.

We now give some definitions.

Definition 3 1. The individually rational attainable allocations set $\mathcal{A}$ is defined by

$$
\mathcal{A}=\left\{\left(x^{i}\right) \in\left(l^{p}(\pi)\right)^{m} \mid \sum_{i=1}^{m} x^{i}=\sum_{i=1}^{m} e^{i} \text { and } U^{i}\left(x^{i}\right) \geq U^{i}\left(e^{i}\right) \text { for all } i\right\} .
$$

2. The individually rational utility set $\mathcal{U}$ is defined by

$$
\mathcal{U}=\left\{\left(v^{1}, v^{2}, \ldots, v^{m}\right) \in \mathbb{R}^{m} \mid \exists x \in \mathcal{A} \text { s.t } U^{i}\left(e^{i}\right) \leq v^{i} \leq U^{i}\left(x^{i}\right) \text { for all } i\right\} .
$$

\section{No-arbitrage condition and existence of equilib- rium}

We will first strengthen the definition of useful vectors introduced by Werner. We then introduce a notion of no-arbitrage price based on these strong useful vectors.

A vector $w$ is strongly useful for agent $i$ if, for any $x^{i} \in X^{i}$, we have

$$
\begin{aligned}
(i) \sum_{s=1}^{\infty} \pi_{s}^{i} u^{i}\left(x_{s}^{i}+\lambda w_{s}\right) & \geq \sum_{s=1}^{\infty} \pi_{s}^{i} u^{i}\left(x_{s}^{i}\right), \forall \lambda \geq 0 \\
\text { and }(i i) \sum_{s=1}^{\infty} \pi_{s}^{i} u^{i}\left(x_{s}^{i}+\lambda w_{s}\right) & >\sum_{s=1}^{\infty} \pi_{s}^{i} u^{i}\left(x_{s}^{i}\right) \text { for some } \lambda>0
\end{aligned}
$$


It is easy to check that a vector $w$ is strongly useful for agent $i$ if, and only if

$$
\forall x^{i} \in X^{i}, \exists \bar{\lambda}>0, \forall \lambda \geq \bar{\lambda}, \sum_{s=1}^{\infty} \pi_{s}^{i} u^{i}\left(x_{s}^{i}+\lambda w_{s}\right)>\sum_{s=1}^{\infty} \pi_{s}^{i} u^{i}\left(x_{s}^{i}\right) .
$$

This condition turns out to be equivalent to

$$
\sum_{s=1}^{\infty} \pi_{s}^{i} u^{i \prime}\left(x_{s}^{i}\right) w_{s}>0, \forall x^{i} \in X^{i}
$$

Following Dana and Le Van [8], we now introduce a no-arbitrage condition.

(NA) There exist $\left(\bar{x}^{i}\right)_{i=1}^{m} \in \Pi_{i} X^{i}$, such that

$$
\lambda^{i} \pi_{s}^{i} u^{i \prime}\left(\bar{x}_{s}^{i}\right)=\lambda^{j} \pi_{s}^{j} u^{j \prime}\left(\bar{x}_{s}^{j}\right)=\lambda^{k} a_{k} \pi_{s}^{k}, \forall s, \forall i \in I_{1}, \forall j \in I_{1}, \forall k \in I_{2},
$$

and

$$
\forall i \in I_{1}, a^{i}<\inf _{s} u^{i \prime}\left(\bar{x}_{s}^{i}\right)<\sup _{s} u^{i \prime}\left(\bar{x}_{s}^{i}\right)<b^{i} .
$$

Observe that if we define a price $p \in l_{+}^{\infty}(\pi)$ by

$$
\forall s, p_{s}=\lambda^{i} \pi_{s}^{i} u^{i \prime}\left(\bar{x}_{s}^{i}\right)=\lambda^{k} a_{k} \pi_{s}^{k}, i \in I_{1}, k \in I_{2}
$$

then we have $p \cdot w>0$ for any strongly useful vector $w$.

Condition (NA) is useful to prove the boundedness of $\mathcal{U}$. That is the statement of the following proposition. The proof is given in Appendix.

Proposition 1 If (NA) holds, then $\mathcal{U}$ is bounded.

The next proposition and its corollary give sufficient conditions to obtain (NA).

Proposition 2 Assume either $u^{i \prime}(-\infty)=+\infty$ for all $i \in I_{1}$ or $u^{i \prime}(+\infty)=0$ for all $i \in I_{1}$. Then (NA) holds if, and only if, $\pi^{i}=\pi^{j}$, for any $i \in I_{2}$, any $j \in I_{2}$.

The following corollary is immediate.

Corollary 1 If $I_{2}=\emptyset$, then (NA) holds if

(i) either $u^{i \prime}(-\infty)=+\infty$ for all $i \in I$,

(ii) or $u^{i \prime}(+\infty)=0$ for all $i \in I$.

In the case of finite number of states, condition (NA) implies compactness of $\mathcal{U}$.

In infinite dimension, with a vector space $L$ as commodity space, Brown and Werner [4], Dana, Le Van and Magnien [10] assume the compactness of $\mathcal{U}$ and get existence of equilibrium with prices in $L^{\prime}$. In our model if the number 
of states is finite, condition (NA) implies compactness of $\mathcal{U}$ and is a sufficient condition for the existence of equilibrium (see e.g. Dana and Le Van [8]).

In this paper, the number of states is infinite, we give an example which shows that (NA) is not sufficient to ensure existence of equilibrium if the conditions in Corollary 1 do not hold. We exhibit a model with two agents. Agent 1 has $a^{1}=0, b^{1}<+\infty$. Agent 2 has $a^{2}>0, b^{2}=+\infty$. The assumptions of Propositions 4 and 6 are clearly not satisfied. We still have (NA) but no equilibrium exists in this model.

Example 1 Consider an economy with two agents $(i=1,2)$, with endowments equal to 0 . The probabilities are equivalent: $\pi_{s}^{1}=\left(\frac{1}{2}\right)^{s}, \pi_{s}^{2}=\frac{1}{S_{\alpha}} \frac{1-\alpha^{s}}{2^{s}}$, where $1<\alpha<1$, and $S_{\alpha}=\sum_{s} \frac{1-\alpha^{s}}{2^{s}}$.

The reward utilities satisfy

$$
\begin{aligned}
u^{1 \prime}(x) & =b^{1}, \forall x \leq 0, \\
u^{1 \prime}(+\infty) & =0, \\
u^{1}(0) & =0, \\
u^{2 \prime}(x) & =a^{2}, \forall x \geq 0, \\
u^{2 \prime}(-\infty) & =+\infty, \\
u^{2}(0) & =0 .
\end{aligned}
$$

There exists $z>0$ with $u^{1 \prime}(z)<b^{1}$. Let $\bar{x}_{s}^{1}=z, \forall s$. Since $u^{2 \prime}(-\infty)=+\infty$, there exists $\bar{x}_{s}^{2}<0$ which satisfies

$$
u^{2 \prime}(0)=\left(1-\alpha^{s}\right) u^{2 \prime}\left(\bar{x}_{s}^{2}\right) .
$$

One can check that

$$
\lambda \pi_{s}^{1} u^{1 \prime}\left(\bar{x}_{s}^{1}\right)=\pi_{s}^{2} u^{2 \prime}\left(\bar{x}_{s}^{2}\right), \forall s,
$$

with $\lambda=\frac{u^{2 \prime}(0)}{u^{1 \prime}(z)} \times \frac{1}{S_{\alpha}}$. Since

$$
\begin{gathered}
0=u^{1 \prime}(+\infty)<u^{1 \prime}(z)=u^{1 \prime}\left(\bar{x}_{s}^{1}\right)<b^{1}, \\
a^{2}=u^{2 \prime}(0)<\frac{u^{2 \prime}(0)}{1-\alpha^{s}}=u^{2 \prime}\left(\bar{x}_{s}^{2}\right)<u^{2 \prime}(-\infty)=+\infty
\end{gathered}
$$

no-arbitrage condition (NA) is satisfied.

We now show that no equilibrium exists. Assume there exists an equilibrium $\left(p,\left(x^{1}, x^{2}\right)\right)$ with $x_{s}^{1}=x_{s}=-x_{s}^{2}$. We have

$$
\begin{aligned}
\forall s, \lambda_{1} \pi_{s}^{1} u^{1 \prime}\left(x_{s}\right) & =\lambda_{2} \pi_{s}^{2} u^{2 \prime}\left(-x_{s}\right), \\
\text { or } \lambda \pi_{s}^{1} u^{1 \prime}\left(x_{s}\right) & =\pi_{s}^{2} u^{2 \prime}\left(-x_{s}\right), \text { with } \lambda=\frac{\lambda_{1}}{\lambda_{2}} .
\end{aligned}
$$


For all s:

$$
\lambda^{\prime} \frac{1}{2^{s}} u^{1 \prime}\left(x_{s}\right)=\frac{1-\alpha^{s}}{2^{s}} u^{2 \prime}\left(-x_{s}\right)
$$

or equivalently

$$
\lambda^{\prime}=\left(1-\alpha^{s}\right) \frac{u^{2 \prime}\left(-x_{s}\right)}{u^{1 \prime}\left(x_{s}\right)}, \forall s
$$

with $\lambda^{\prime}=\lambda S_{\alpha}$. Since $\sum_{s} p_{s} x_{s}=0$ and $p_{s}>0$ for any $s$, one must have $s_{0}$ with $x_{s_{0}} \leq 0$. In this case

$$
\lambda^{\prime}=\left(1-\alpha^{s_{0}}\right) \frac{a^{2}}{b^{1}}
$$

and for any $s \neq s_{0}, x_{s}>0$. We then obtain

$$
\frac{u^{2 \prime}\left(-x_{s_{0}+1}\right)}{u^{1 \prime}\left(x_{s_{0}+1}\right)}>\frac{u^{2 \prime}(0)}{u^{1 \prime}(0)}=\frac{a^{2}}{b^{1}}
$$

since $x_{s_{0}+1}>0$. Now, because $1-\alpha^{s_{0}+1}>1-\alpha^{s_{0}}$ we obtain, on the one hand:

$$
\lambda^{\prime}=\left(1-\alpha^{s_{0}+1}\right) \frac{u^{2 \prime}\left(-x_{s_{0}+1}\right)}{u^{1 \prime}\left(x_{s_{0}+1}\right)}>\left(1-\alpha^{s_{0}}\right) \frac{u^{2 \prime}\left(-x_{s_{0}+1}\right)}{u^{1 \prime}\left(x_{s_{0}+1}\right)}>\left(1-\alpha^{s_{0}}\right) \frac{a^{2}}{b^{1}}
$$

and on the other hand

$$
\lambda^{\prime}=\left(1-\alpha^{s_{0}}\right) \frac{a^{2}}{b^{1}}
$$

which is a contradiction. Then there exists no equilibrium.

We now state our main results. Their proofs are given in Appendix. The ideas are to prove that the set $\mathcal{A}$ is $l^{1}(\pi)$-compact and hence the set $\mathcal{U}$ is also compact when $b^{i}=+\infty$ for all $i$, and when $a^{i}=0$ for every $i$ and $b^{i}<+\infty$ for some $i$, the set $\mathcal{A}$ is not necessary $l^{1}(\pi)$-compact but the set $\mathcal{U}$ is still compact.

Theorem 1 Suppose one of these two conditions holds:

(i) $a^{i}=0$ for every $i$.

(ii) $b^{i}=+\infty$ for every $i$.

Then there exists an equilibrium with equilibrium price in $l^{q}(\pi)$ with $\frac{1}{p}+\frac{1}{q}=$ $1,1 \leq p \leq+\infty$.

Theorem 2 Suppose that $I_{2} \neq \emptyset$. Suppose that these two conditions hold:

(i) $a^{i}=0$ for every $i \in I_{1}$.

(ii) $b^{i}=+\infty$ for every $i \in I_{1}$.

Then we have

$$
(N A) \Leftrightarrow \exists \text { Equilibrium. }
$$

The equilibrium price is in $l^{q}(\pi)$ with $\frac{1}{p}+\frac{1}{q}=1,1 \leq p \leq+\infty$. 
We can be surprised that in presence of risk-neutral agents we have to impose $u^{i \prime}(+\infty)=0$ and $u^{i \prime}(-\infty)=+\infty$ for any agent $i \in I_{1}$. We give two examples with two agents, one of them is risk neutral while the second one is risk averse. In Example 2, the risk averse agent has the marginal utility at $-\infty$ equal to $+\infty$. In Example 3, her the marginal utility at $+\infty$ is 0 . In both examples, there exists no equilibrium.

Example 2 Consider an economy with two agents $(i=1,2)$, with endowments equal to 0 . The probabilities are equivalent: $\pi_{s}^{1}=\left(\frac{1}{2}\right)^{s}, \pi_{s}^{2}=\frac{1}{S_{\alpha}} \frac{1-\alpha^{s}}{2^{s}}$, where $1<\alpha<1$, and $S_{\alpha}=\sum_{s} \frac{1-\alpha^{s}}{2^{s}}$. Agent 1 is risk neutral.

The reward utilities satisfy

$$
\begin{aligned}
u^{1 \prime}(x) & =1, \forall x \in \mathbb{R}, \\
u^{2 \prime}(x) & =a^{2}, \forall x \geq 0, \\
u^{2 \prime}(-\infty) & =+\infty, \\
u^{2 \prime}(x) & >a^{2}, \forall x<0 .
\end{aligned}
$$

Assume there exists an equilibrium $\left(p,\left(x^{1}, x^{2}\right)\right)$. Then $x_{s}^{1}=-x_{s}^{2}=x_{s}$ for any s. There exists $\lambda>0$ such that

$$
\begin{aligned}
\frac{\lambda}{2^{s}} u^{1 \prime}\left(x_{s}\right) & =\frac{1-\alpha^{s}}{S_{\alpha} 2^{s}} u^{2 \prime}\left(-x_{s}\right) \forall s \\
\Leftrightarrow u^{2 \prime}\left(-x_{s}\right) & =\lambda \frac{S_{\alpha}}{1-\alpha^{s}} \forall s .
\end{aligned}
$$

Since $\sum_{s} p_{s} x_{s}=0$ and $p_{s}>0$ for all $s$, there exists $x_{s_{0}} \leq 0$, i.e $-x_{s_{0}} \geq 0$. Then

$$
a^{2}=\lambda \frac{S_{\alpha}}{1-\alpha^{s_{0}}}
$$

and $\forall s \neq s_{0}, x_{s}>0$. Hence $u^{2 \prime}\left(-x_{s_{0}+1}\right)>a^{2}$. This implies

$$
\lambda \frac{S_{\alpha}}{1-\alpha^{s_{0}+1}}>\lambda \frac{S_{\alpha}}{1-\alpha^{s_{0}}} \Rightarrow \alpha^{s_{0}+1}>\alpha^{s_{0}} .
$$

A contradiction.

Example 3 Agent 2 is risk neutral. The reward utilities satisfy

$$
\begin{aligned}
u^{1 \prime}(x) & =b^{1}, \forall x \leq 0, \\
u^{1 \prime}(+\infty) & =0, \\
u^{1 \prime}(x) & <b^{1}, \forall x>0, \\
u^{2 \prime}(x) & =1, \forall x \in \mathbb{R} .
\end{aligned}
$$


Assume there exists an equilibrium $\left(p,\left(x^{1}, x^{2}\right)\right)$. Then $x_{s}^{1}=-x_{s}^{2}=x_{s}$ for any s. There exists $\lambda>0$ such that

$$
u^{1 \prime}\left(x_{s}\right)=\lambda \frac{1-\alpha^{s}}{S_{\alpha}} \forall s .
$$

Since $\sum_{s} p_{s} x_{s}=0$ and $p_{s}>0$ for all $s$, there exists $x_{s_{0}} \leq 0$, i.e $-x_{s_{0}} \geq 0$. Then

$$
b^{1}=\lambda \frac{1-\alpha^{s_{0}}}{S_{\alpha}}
$$

and $\forall s \neq s_{0}, x_{s}>0$. Hence $u^{1 \prime}\left(x_{s_{0}+1}\right)<b^{1}$. This implies

$$
\lambda \frac{1-\alpha^{s_{0}+1}}{S_{\alpha}}<\lambda \frac{1-\alpha^{s_{0}}}{S_{\alpha}} \Rightarrow \alpha^{s_{0}+1}>\alpha^{s_{0}} .
$$

A contradiction.

Remark 1 When there are short-sales constraints, the allocation set $\mathcal{A}$ is compact in $l^{p}(\pi)$ for any $p$. Indeed, suppose we have short-sales constraints $x_{s}^{i} \geq c_{s}$ for every $i, s$, with $c=\left(c_{1}, c_{2}, \ldots, c_{s}, \ldots\right) \in l^{p}(\pi)$. We also have for every $\left(x^{1}, x^{2}, \ldots, x^{m}\right)$ which belongs to $\mathcal{A}$ :

$$
x_{s}^{i}=e_{s}-\sum_{j \neq i} x_{s}^{j} \leq e_{s}-\sum_{j \neq i} c_{s} \leq\left|e_{s}\right|+(m-1)\left|c_{s}\right| .
$$

$\mathcal{A}$ is hence compact for the product topology. Suppose that the sequence $\left(x^{1}(n), x^{2}(n), \ldots, x^{m}(n)\right)$ belong to $\mathcal{A}$ converges to $\left(\bar{x}^{1}, \bar{x}^{2}, \ldots, \bar{x}^{m}\right)$ for the product topology when $n$ tends to infinity. We have for every $i$,

$$
c_{s} \leq \bar{x}_{s}^{i} \leq\left|e_{s}\right|+(m-1)\left|c_{s}\right| .
$$

This implies that $\left(\bar{x}^{1}, \bar{x}^{2}, \ldots, \bar{x}^{m}\right) \in l^{p}(\pi)$. The set of individually rational allocations $\mathcal{A}$ is compact in $l^{p}(\pi)$. That implies the compactness of $\mathcal{U}$ and the existence of a quasi-equilibrium. This explain why in our paper we assume no short-sales constraints.

Concluding remarks $(a)$ In our model with an infinitely countable number of states of nature, the sufficient conditions for the existence of an equilibrium are:

(i) Either the marginal utilites at $+\infty$ equal zero for all the agents,

(ii) or the marginal utilites at $-\infty$ equal $+\infty$ for all the agents.

We give examples where agents do not satisfy the conditions above. Their utility functions satisfy the no-arbitrage condition à la Werner. However there is no equilibrium. 
(b) Our sufficient conditions differ from the ones in Le Van and Truong Xuan (Assumption H4) [16], and Daher, Martins-da-Rocha, Vailakis, [11] (Assumption S4). Put in our model, these conditions are stronger than ours. Indeed, they imply, for our model, that for all the agents, the marginal utilities at $+\infty$ equal zero and the marginal utilities at $-\infty$ equal $+\infty$.

\section{Appendix}

\section{Proof of Proposition 1}

Take any NA-price $p$. There exists $\left(\bar{x}^{i}\right), \lambda^{1}, \lambda^{2}, \ldots, \lambda^{m}$ positive such that for all $i, s: p_{s}=\lambda^{i} \pi_{s}^{i} u^{i \prime}\left(\bar{x}_{s}^{i}\right)$.

(i) For $i, j \in I_{2}$ we have $\lambda^{i} a^{i} \pi_{s}^{i}=\lambda^{j} a^{j} \pi_{s}^{j}, \forall s$. Take the sum we have:

$$
\sum_{s=1}^{\infty} \lambda^{i} a^{i} \pi_{s}^{i}=\sum_{s=1}^{\infty} \lambda^{j} a^{j} \pi_{s}^{j} .
$$

This implies $\lambda^{i} a^{i}=\lambda^{j} a^{j}$, hence $\pi^{i}=\pi^{j}$.

(ii) We firstly prove that there exists $C>0$ such that:

$$
\sum_{i \in I_{1}} \sum_{s=1}^{\infty} \pi_{s}^{i}\left|x_{s}^{i}\right| \leq C .
$$

Define $e^{\prime}=\bar{e}-\sum_{i \in I_{2}} x^{i}=\sum_{i \in I_{1}} x^{i} \in l^{1}(\pi)$.

For $i \in I_{1}$, since $a^{i}<\inf _{s} u^{i \prime}\left(\bar{x}_{s}^{i}\right) \leq \sup _{s} u^{i \prime}\left(\bar{x}_{s}^{i}\right)<b^{i}$, we have $\bar{x}^{i} \in l^{\infty}(\pi)$. Observe that $p \in l^{\infty}(\pi)$.

Choose $\eta>0$ such that

$$
a^{i}<u^{i \prime}\left(\bar{x}_{s}^{i}\right)(1+\eta)<b^{i} \text { for }
$$

for all $i \in I_{1}$. Then we define the price $q$ as follows: $\forall i, j \in I_{1}$,

$$
q_{s}=p_{s}(1+\eta)=\lambda^{i} \pi_{s}^{i} u^{i \prime}\left(\bar{x}_{s}^{i}\right)(1+\eta)=\lambda^{j} \pi_{s}^{j} u^{j \prime}\left(\bar{x}_{s}^{j}\right)(1+\eta) .
$$

It follows that, for each $i \in I_{1}$, there exist $\bar{z}^{i}$ such that $\forall s, q_{s}=\lambda^{i} \pi_{s}^{i} u^{i \prime}\left(\bar{z}_{s}^{i}\right)$. Observe that $a^{i}<\inf _{s} u^{i \prime}\left(z_{s}^{i}\right) \leq \sup _{s} u^{i \prime}\left(z_{s}^{i}\right)<b^{i}$, so $\bar{z}^{i} \in l^{\infty}(\pi)$. Observe also that $\forall s, p_{s}<q_{s}$.

Denote

$$
\begin{aligned}
& x^{+}:=\left\{\begin{array}{r}
x \text { if } x>0, \\
0 \text { if } x \leq 0
\end{array}\right. \\
& x^{-}:=\left\{\begin{array}{r}
-x \text { if } x<0, \\
0 \text { if } x \geq 0
\end{array}\right.
\end{aligned}
$$

Observe that $x=x^{+}-x^{-},|x|=x^{+}+x^{-}$and $u(x)=u\left(x^{+}\right)+u\left(-x^{-}\right)-u(0)$. 
Now we fix $N \in \mathbb{N}$. For $i \in I_{1}$, from the concavity of the utility function $u^{i}$ we have

$$
\begin{aligned}
\lambda^{i} \sum_{s=1}^{N} \pi_{s}^{i} u^{i}\left(\bar{x}_{s}^{i}\right)-\lambda^{i} \sum_{s=1}^{N} \pi_{s}^{i} u^{i}\left(x_{s}^{i+}\right) & \geq \lambda^{i} \sum_{s=1}^{N} \pi_{s}^{i} u^{i \prime}\left(\bar{x}_{s}^{i}\right)\left(\bar{x}_{s}^{i}-x_{s}^{i+}\right), \\
\lambda^{i} \sum_{s=1}^{N} \pi_{s}^{i} u^{i}\left(\bar{z}_{s}^{i}\right)-\lambda^{i} \sum_{s=1}^{N} \pi_{s}^{i} u^{i}\left(-x_{s}^{i-}\right) & \geq \lambda^{i} \sum_{s=1}^{N} \pi_{s}^{i} u^{i \prime}\left(\bar{z}_{s}^{i}\right)\left(\bar{z}_{s}^{i}+x_{s}^{i-}\right) .
\end{aligned}
$$

Therefore,

$$
\begin{aligned}
\lambda^{i} \sum_{s=1}^{N} \pi_{s}^{i} u^{i \prime}\left(\bar{z}_{s}^{i}\right) x_{s}^{i-} \leq & \lambda^{i} \sum_{s=1}^{N} \pi_{s}^{i}\left[u^{i}\left(\bar{z}_{s}^{i}\right)+u^{i}\left(\bar{x}_{s}^{i}\right)-u^{i}\left(x_{s}^{i+}\right)-u^{i}\left(-x_{s}^{i-}\right)\right] \\
& -\lambda^{i} \sum_{s=1}^{N} \pi_{s}^{i} u^{i \prime}\left(\bar{z}_{s}^{i}\right) \bar{z}_{s}^{i}+\lambda^{i} \sum_{s=1}^{N} \pi_{s}^{i} u^{i \prime}\left(\bar{x}_{s}^{i}\right) x_{s}^{i+}-\lambda^{i} \sum_{s=1}^{N} \pi_{s}^{i} u^{i \prime}\left(\bar{x}_{s}^{i}\right) \bar{x}_{s}^{i} .
\end{aligned}
$$

Define $U_{N}^{i}(x):=\sum_{s=1}^{N} \pi_{s}^{i} u^{i}\left(x_{s}\right)$. Note that $\lim _{N \rightarrow \infty} U_{N}^{i}(x)=U^{i}(x)$. The above inequality implies

$$
\begin{aligned}
\sum_{s=1}^{N} q_{s} x_{s}^{i-} & \leq \lambda^{i}\left[U_{N}^{i}\left(\bar{z}^{i}\right)+U_{N}^{i}\left(\bar{x}^{i}\right)-U_{N}^{i}\left(x^{i}\right)-U_{N}^{i}(0)\right] \\
& -\sum_{s=1}^{N} q_{s} \bar{z}_{s}^{i}+\sum_{s=1}^{N} p_{s} x_{s}^{i+}-\sum_{s=1}^{N} p_{s} \bar{x}_{s}^{i} \\
& \leq \lambda^{i}\left[U_{N}^{i}\left(\bar{z}^{i}\right)+U_{N}^{i}\left(\bar{x}^{i}\right)-U_{N}^{i}\left(x^{i}\right)-U_{N}^{i}(0)\right] \\
& -\sum_{s=1}^{N} p_{s}^{i} \bar{x}_{s}^{i}-\sum_{s=1}^{N} q_{s} \bar{z}_{s}^{i}+\sum_{s=1}^{N} p_{s} x_{s}^{i+} \\
& =C_{N}^{i}+\sum_{s=1}^{N} p_{s} x_{s}^{i+}
\end{aligned}
$$

where $C_{N}^{i}=\lambda^{i}\left[U_{N}^{i}\left(\bar{z}^{i}\right)+U_{N}^{i}\left(\bar{x}^{i}\right)-U_{N}^{i}\left(x^{i}\right)-U_{N}^{i}(0)\right]-\sum_{s=1}^{N} p_{s}^{i} \bar{x}_{s}^{i}-\sum_{s=1}^{N} q_{s} \bar{z}_{s}^{i}$.

Observe that since $\bar{x}^{i}$ et $\bar{z}^{i}$ belong to $l^{\infty}(\pi)$, the $\operatorname{limit}_{\lim _{N}} C_{N}^{i}$ exists.

Hence, $\forall i$

$$
\sum_{s=1}^{N}\left(q_{s}-p_{s}\right) x_{s}^{i-} \leq C_{N}^{i}+\sum_{s=1}^{N} p_{s} x_{s}^{i}
$$

Thus, we have

$$
\sum_{i \in I_{1}} \sum_{s=1}^{N}\left(q_{s}-p_{s}\right) x_{s}^{i-} \leq \sum_{i \in I_{1}} C_{N}^{i}+\sum_{i \in I_{1}} \sum_{s=1}^{N} p_{s} x_{s}^{i}=\sum_{i \in I_{1}} C_{N}^{i}+\sum_{s=1}^{N} p_{s} e_{s}^{\prime} .
$$

Since $e^{\prime} \in l^{1}(\pi), \sum_{i \in I_{1}} C_{N}^{i}+\sum_{s=1}^{N} p_{s} e_{s}^{\prime}$ converges. Now let $N$ tends to infinity. Notice that $U_{N}^{i}(x) \rightarrow U^{i}(x)$ for all $x$, and recall that $U^{i}\left(x^{i}\right) \geq U^{i}\left(e^{i}\right)$, 
with $\bar{x}^{i}, \bar{z}^{i} \in l^{\infty}(\pi)$. We then have

$$
\limsup _{N \rightarrow \infty} C_{N}^{i} \leq \lambda^{i}\left[U^{i}\left(\bar{z}^{i}\right)+U^{i}\left(\bar{x}^{i}\right)-U^{i}\left(e^{i}\right)-U^{i}(0)\right]-\sum_{s=1}^{\infty} q_{s} \bar{z}_{s}^{i}-\sum_{s=1}^{\infty} p_{s} \bar{x}_{s}^{i}=: C^{i} .
$$

Thus,

$$
\sum_{i \in I_{1}} \sum_{s=1}^{\infty}\left(q_{s}-p_{s}\right) x_{s}^{i-} \leq \sum_{i \in I_{1}} C^{i}+\sum_{s=1}^{\infty} p_{s} e_{s}^{\prime}=: C_{1}+\sum_{s=1}^{\infty} p_{s} e_{s}^{\prime}
$$

We also have

$$
\sum_{i \in I_{1}} \sum_{s=1}^{\infty}\left(q_{s}-p_{s}\right)\left(x_{s}^{i+}-x_{s}^{i-}\right)=\sum_{i \in I_{1}} \sum_{s=1}^{\infty}\left(q_{s}-p_{s}\right) x_{s}^{i}=\sum_{s=1}^{\infty}\left(q_{s}-p_{s}\right) e_{s}^{\prime}
$$

which implies

$$
\begin{aligned}
\sum_{i \in I_{1}} \sum_{s=1}^{\infty}\left(q_{s}-p_{s}\right) x_{s}^{i+} & =\sum_{s=1}^{\infty}\left(q_{s}-p_{s}\right) e_{s}^{\prime}+\sum_{i \in I_{1}} \sum_{s=1}^{\infty}\left(q_{s}-p_{s}\right) x_{s}^{i-} \\
& \leq C_{1}+\sum_{s=1}^{\infty} p_{s} e_{s}^{\prime}+\sum_{s=1}^{\infty}\left(q_{s}-p_{s}\right) e_{s}^{\prime} \\
& =C_{1}+\sum_{s=1}^{\infty} q_{s} e_{s}^{\prime} .
\end{aligned}
$$

Thus for $i \in I_{1}$

$$
\begin{aligned}
\sum_{s=1}^{\infty}\left(q_{s}-p_{s}\right)\left|x_{s}^{i}\right| & \leq 2 C_{1}+\sum_{s=1}^{\infty}\left(p_{s}+q_{s}\right) e_{s}^{\prime} \\
& =2 C_{1}+(2+\eta) \sum_{s=1}^{\infty} p_{s} e_{s}^{\prime} \\
& =2 C_{1}+(2+\eta) \sum_{s=1}^{\infty} p_{s} \bar{e}_{s}-(2+\eta) \sum_{i \in I_{2}} \sum_{s=1}^{\infty} p_{s} x_{s}^{i} \\
& =2 C_{1}+(2+\eta) \sum_{s=1}^{\infty} p_{s} \bar{e}_{s}-(2+\eta) \sum_{i \in I_{2}} \lambda^{i} a^{i} \sum_{s=1}^{\infty} \pi_{s}^{i} x_{s}^{i} \\
& =2 C_{1}+(2+\eta) \sum_{s=1}^{\infty} p_{s} \bar{e}_{s}-(2+\eta) \sum_{i \in I_{2}} \lambda^{i} U^{i}\left(x^{i}\right) \\
& \leq 2 C_{1}+(2+\eta) \sum_{s=1}^{\infty} p_{s} \bar{e}_{s}-(2+\eta) \sum_{i \in I_{2}} \lambda^{i} U^{i}\left(e^{i}\right) \\
& =C^{2},
\end{aligned}
$$

then

$$
\eta \sum_{s=1}^{\infty} p_{s}\left|x_{s}^{i}\right| \leq C^{2}
$$


Let $\mu_{i}:=\inf _{s} u^{i \prime}\left(\bar{x}_{s}^{i}\right)>0$, and $\mu:=\min _{i} \mu_{i}$. Then $\sum_{s=1}^{\infty} p_{s}\left|x_{s}^{i}\right| \geq \mu \sum_{s=1}^{\infty} \pi_{s}^{i}\left|x_{s}^{i}\right|$ which implies for all $i \in I_{1}$

$$
\sum_{s=1}^{\infty} \pi_{s}^{i}\left|x_{s}^{i}\right| \leq D_{1}
$$

with $D_{1}=C^{2} /(\eta \mu)$.

For $I_{2}$ we have:

$$
\begin{aligned}
\sum_{s=1}^{\infty} \pi_{s}^{I}\left|\sum_{i \in I_{2}} x_{s}^{i}\right| & \leq \sum_{s=1}^{\infty} \pi_{s}^{I}\left|\bar{e}_{s}\right|+\sum_{i \in I_{1}} \sum_{s=1}^{\infty} \pi_{s}^{i}\left|x_{s}^{i}\right| \\
& \leq D_{2}
\end{aligned}
$$

with $D_{2}=\sum_{s=1}^{\infty} \pi_{s}^{I}\left|\bar{e}_{s}\right|+\left|I_{1}\right| D_{1}$.

We take $C=\max \left\{D_{1}, D_{2}\right\}$.

(iii) By Jensen inequality, the utility set $\mathcal{U}$ is bounded.

\section{Proof of Proposition 2}

(i) Consider the case where $I_{2}=\emptyset$.

(a) Assume $u^{i \prime}(-\infty)=+\infty$, for any $i$. Let $z$ satisfy $a^{1}<u^{1 \prime}(z)$. For $i>1$, let

$$
\zeta_{s}^{i}=\frac{\pi_{s}^{1}}{\pi_{s}^{i}} u^{1 \prime}(z), \forall s
$$

Then $\frac{1}{h} u^{1 \prime}(z) \geq \zeta_{s}^{i} \geq h u^{1 \prime}(z)$. One can find $\lambda^{i}$ s.t. $\frac{\zeta_{s}^{i}}{\lambda^{i}} \geq \alpha^{i}>a^{i}$. Define

$$
u^{i \prime}\left(x_{s}^{i}\right)=\frac{\zeta_{s}^{i}}{\lambda^{i}}, \forall s .
$$

Then

$$
a^{i}<\alpha^{i} \leq u^{i \prime}\left(x_{s}^{i}\right) \leq \frac{1}{\lambda^{i} h} u^{1 \prime}(z), \forall s
$$

Since $b^{i}=+\infty$, we have $x^{i} \in l^{\infty}(\pi)$. Obviously

$$
\lambda^{i} \pi_{s}^{i} u^{i \prime}\left(x_{s}^{i}\right)=\pi_{s}^{1} u^{1 \prime}(z), \forall s .
$$

(b) Assume $u^{i \prime}(+\infty)=0$ for all $i$. Let $z$ satisfy $0<u^{1 \prime}(z)<b^{1}$. Define $\zeta_{s}^{i}$ as before. We have $\zeta_{s}^{i} \leq \frac{1}{h} u^{1 \prime}(z)$. Choose $\lambda^{i}$ s.t.

$\frac{\zeta_{s}^{i}}{\lambda^{i}} \leq \beta^{i}<b^{i}$. Then define $x_{s}^{i}$ as before. Using the same arguments, we have

$$
\lambda^{i} \pi_{s}^{i} u^{i \prime}\left(x_{s}^{i}\right)=\pi_{s}^{1} u^{1 \prime}(z), \forall s .
$$

(ii) Now we consider the case where $I_{2} \neq \emptyset$. If (NA) holds, let $p$ be a (NA)-price. There exists $\left(\bar{x}^{i}\right), \lambda^{1}, \lambda^{2}, \ldots, \lambda^{m}$ positive such that for all $i, s$ we have $p_{s}=\lambda^{i} \pi_{s}^{i} u^{i \prime}\left(\bar{x}_{s}^{i}\right)$.

For $i, j \in I_{2}$ we have $\lambda^{i} a^{i} \pi_{s}^{i}=\lambda^{j} a^{j} \pi_{s}^{j}, \forall s$. Taking the sum we have:

$$
\sum_{s=1}^{\infty} \lambda^{i} a^{i} \pi_{s}^{i}=\sum_{s=1}^{\infty} \lambda^{j} a^{j} \pi_{s}^{j} .
$$


This implies $\lambda^{i} a^{i}=\lambda^{j} a^{j}$, hence $\pi^{i}=\pi^{j}$.

Conversely, assume that $\pi^{k}=\pi^{l}=\pi^{\prime}, \forall k \in I_{2}, \forall l \in I_{2}$. Assume $1 \in I_{2}$. For $i \in I_{2}, i \neq 1$, choose $\lambda^{i}$ such that $\lambda^{i} a^{i}=a^{1}$.

Consider the case $u^{i \prime}(-\infty)=+\infty, \forall i \in I_{1}$.

For $i \in I_{1}$, define $\zeta_{s}^{i}$ :

$$
\frac{1}{h} a^{1} \geq \zeta_{s}^{i}=\frac{a^{1} \pi_{s}^{1}}{\pi_{s}^{i}} \geq a^{1} h
$$

There exists $\lambda^{i}$ s.t.

$$
\frac{\zeta_{s}^{i}}{\lambda^{i}} \geq \alpha^{i}>a^{i}
$$

and

$$
a^{i}<u^{i \prime}\left(x_{s}^{i}\right)=\frac{\zeta_{s}^{i}}{\lambda^{i}} \leq \frac{1}{h} a_{1} .
$$

Since $b^{i}=+\infty$, we have $x^{i} \in l^{\infty}(\pi)$. Obviously

$$
\lambda^{i} \pi_{s}^{i} u^{i \prime}\left(x_{s}^{i}\right)=\pi_{s}^{1} u^{1 \prime}(z), \forall s .
$$

We use the same argument as before if $u^{i \prime}(+\infty)=0, \forall i \in I_{1}$.

\section{Proofs of Theorems 1, 2}

The proofs require many intermediary steps.

Claims 1, 2 and 3 are required for the proof of Claim 4.

Claim 1 Assume AO. Our model has an equilibrium if we add the assumption that $\mathcal{U}$ is compact. If $X^{i}$ is $l^{p}(\pi)$ with $1 \leq p<+\infty$ then the equilibrium price $p^{*}$ is in $l^{q}(\pi), \frac{1}{p}+\frac{1}{q}=1$. If $p=+\infty$, then $p^{*} \in l^{1}(\pi)$.

Proof: Since $\mathcal{U}$ is compact and $X^{i}$ is $l^{p}(\pi)$ there exists an equilibrium $\left(\left(x^{* i}\right), p^{*}\right)$ (see Dana and al [9]) with $x^{* i} \in l^{p}(\pi)$.

When $1 \leq p<+\infty$, the price $p^{*}$ belongs to $l^{q}(\pi)$. When $p=\infty$ we will show that the equilibrium price belongs to $l^{1}(\pi)$. The equilibrium price can be written as $p^{*}+\phi$ where $p^{*} \in l^{1}(\pi)$ and $\phi$ is a purely finitely additive function. For any $i$, the equilibrium allocation $x^{* i}$ solves the problem:

$$
\begin{aligned}
& \max \sum_{s=1}^{\infty} \pi_{s}^{i} u^{i}\left(x_{s}^{i}\right), \\
& \text { s.t. } \sum_{s=1}^{\infty} p_{s}^{*} x_{s}^{i}+\phi\left(x^{i}\right)=\sum_{s=1}^{\infty} p_{s}^{*} e_{s}^{i}+\phi\left(e^{i}\right) .
\end{aligned}
$$

From Theorem V.3.1, page 91, in Arrow-Hurwicz-Uzawa in [2], for any $i$, there exists $\zeta_{i}>0$ s.t.

$$
\sum_{s=1}^{\infty} \pi_{s}^{i} u^{i}\left(x_{s}^{* i}\right)-\zeta_{i}\left(\sum_{s=1}^{\infty} p_{s}^{*} x_{s}^{* i}+\phi\left(x^{* i}\right)\right) \geq \sum_{s=1}^{\infty} \pi_{s}^{i} u^{i}\left(x_{s}\right)-\zeta_{i}\left(\sum_{s=1}^{\infty} p_{s}^{*} x_{s}+\phi\left(x^{i}\right)\right) .
$$


Suppose that $\phi \neq 0$. Since $\phi \geq 0$, then $\phi(\mathbf{1})>0$, with $\mathbf{1}=(1,1,1, \ldots)$. Define $x^{i}(N)$ as:

$$
\begin{gathered}
x_{s}^{i}(N)=x_{s}^{* i} \text { with } s=1,2, \ldots, N, \\
x_{s}^{i}(N)=x_{s}^{* i}-1 \text { with } s \geq N+1 .
\end{gathered}
$$

Observe that $x^{i}(N) \in l^{\infty}(\pi)$. We have:

$$
\begin{aligned}
& \sum_{s=1}^{\infty} \pi_{s}^{i} u^{i}\left(x_{s}^{* i}\right)-\zeta_{i}\left(\sum_{s=1}^{\infty} p_{s}^{*} x_{s}^{* i}+\phi\left(x^{* i}\right)\right) \geq \sum_{s=1}^{\infty} \pi_{s}^{i} u^{i}\left(x_{s}^{i}(N)\right)-\zeta_{i}\left(\sum_{s=1}^{\infty} p_{s}^{*} x_{s}^{i}(N)+\phi\left(x^{i}(N)\right)\right) \\
& \Rightarrow \\
& \sum_{s \geq N+1} \pi_{s}^{i} u^{i}\left(x_{s}^{* i}\right)-\zeta_{i}\left(\sum_{s \geq N+1} p_{s}^{*} x_{s}^{* i}+\phi\left(x^{* i}\right)\right) \geq \sum_{s \geq N+1} \pi_{s}^{i} u^{i}\left(x_{s}^{* i}-1\right)-\zeta_{i}\left(\sum_{s \geq N+1} p_{s}^{*}\left(x_{s}^{* i}-1\right)+\phi\left(x^{* i}-\mathbf{1}\right)\right) \\
& \Rightarrow \\
& \sum_{s \geq N+1} \pi_{s}^{i} u^{i}\left(x_{s}^{i *}\right)-\sum_{s \geq N+1} \pi_{s}^{i} u^{i}\left(x_{s}^{* i}-1\right)-\zeta^{i} \sum_{s \geq N+1} p_{s}^{*} \geq \zeta^{i}\left(\phi\left(x^{* i}\right)-\phi\left(x^{* i}-\mathbf{1}\right)\right)=\zeta^{i} \phi(\mathbf{1}) .
\end{aligned}
$$

Let $N \rightarrow \infty$, the left-hand-side converges to 0 . This implies $\phi(\mathbf{1}) \leq 0$ : a contradiction. Hence $\phi=0$.

Claim $2 A$ closed, bounded set $B$ in $l^{1}(\pi)$ is compact if and only if $B$ satisfies the following property: For all $\epsilon>0$, there exists $N \in \mathbb{N}$ such that for all $x \in B$ we have

$$
\sum_{s \geq N} \pi_{s}\left|x_{s}\right|<\epsilon
$$

Proof: Suppose that $B$ is compact and there exists a subsequence $\{x(n)\}_{n}$ of $B, \epsilon>0$ such that

$$
\sum_{s=n}^{\infty} \pi_{s}\left|x_{s}(n)\right|>\epsilon, \forall n
$$

Without loss of generality, we can assume that $x(n)$ converges to $x$ in $l^{1}(\pi)$ or $\|x(n)-x\|_{l^{1}(\pi)} \rightarrow 0$.

By choosing $N$ large enough such that $\|x(n)-x\|_{l^{1}(\pi)}<\frac{\epsilon}{2}$ and $\sum_{s \geq n} \pi_{s}\left|x_{s}\right|<$ $\frac{\epsilon}{2}$ for all $n \geq N$. And for all $n \geq N$, we have

$$
\begin{aligned}
\sum_{s=n}^{\infty} \pi_{s}\left|x_{s}\right| & \geq \sum_{s=n}^{\infty} \pi_{s}\left|x_{s}(n)\right|-\sum_{s=n}^{\infty} \pi_{s}\left|x_{s}(n)-x_{s}\right| \\
& >\epsilon-\frac{\epsilon}{2}=\frac{\epsilon}{2} .
\end{aligned}
$$

A contradiction. 
Now we suppose that for any $\epsilon>0$, there exists $N$ such that $\forall x \in B$, $\sum_{s=N}^{\infty} \pi_{s}\left|x_{s}\right|<\epsilon$.

We have to prove that for any sequence $x(n) \in B$, there exists a convergent subsequence of $x(n)$ in $l^{1}(\pi)$.

Since $B$ is bounded in $l^{1}(\pi)$, there exists $a>0$ such that, $\forall x \in B, \sum_{s \geq 1} \pi_{s}\left|x_{s}\right| \leq$ $a$.

Since $\{x(n)\}_{n}$ belong to a compact set for the product topology, there exists a subsequence $\left\{x\left(n_{k}\right)\right\}_{k}$ which converges to $\bar{x}$ for the product topology. In particular for all $s, x_{s}\left(n_{k}\right)$ converges to $\bar{x}_{s}$ when $k \rightarrow \infty$.

Fix $\epsilon>0$. We will prove that for $k, l$ big enough, $\left\|x\left(n_{k}\right)-x\left(n_{l}\right)\right\|_{l^{1}(\pi)}<\epsilon$. Choose $N>0$ such that for all $x \in B, \sum_{s \geq N} \pi_{s}\left|x_{s}\right|<\frac{\epsilon}{4}$. Choose $M$ such that for all $n_{k}>M$ we have $\sum_{s=1}^{M} \pi_{s}\left|x_{s}\left(n_{k}\right)-\bar{x}_{s}\right|<\frac{\epsilon}{4}$. For all $n_{k} \geq N_{0}, n_{l} \geq N_{0}$ where $N_{0}=\max \{N, M\}$ we have $\sum_{s=1}^{N_{0}} \pi_{s}\left|x_{s}\left(n_{k}\right)-x_{s}\left(n_{l}\right)\right| \leq \sum_{s=1}^{N_{0}} \pi_{s} \mid x_{s}\left(n_{k}\right)-$ $\bar{x}_{s}\left|+\sum_{s=1}^{N_{0}} \pi_{s}\right| x_{s}\left(n_{k}\right)-\bar{x}_{s} \mid<\frac{\epsilon}{2}$.

Then

$$
\begin{aligned}
\sum_{s \geq 1} \pi_{s}\left|x_{s}\left(n_{k}\right)-x_{s}\left(n_{l}\right)\right| & \leq \sum_{s=1}^{N_{0}-1} \pi_{s}\left|x_{s}\left(n_{k}\right)-x_{s}\left(n_{l}\right)\right|+\sum_{s \geq N_{0}} \pi_{s}\left|x_{s}\left(n_{k}\right)\right|+\sum_{s \geq N_{0}} \pi_{s}\left|x_{s}\left(n_{l}\right)\right| \\
& <\epsilon
\end{aligned}
$$

Hence $\left\{x\left(n_{k}\right)\right\}_{k}$ is a Cauchy sequence, then it converges in $l^{1}(\pi)$ topology. So $B$ is compact in $l^{1}(\pi)$ topology.

The following claim is a corollary of Claim 2 .

Claim 3 1. A closed, bounded set $B$ in $l^{1}(\pi)$ is compact for $l^{1}(\pi)$-topology if and only if it is compact for the weak topology $\left.\sigma\left(l^{1}(\pi)\right), l^{\infty}(\pi)\right)$.

2. A closed, bounded set $B$ in $l^{p}(\pi), p>1$ is compact for $l^{1}(\pi)$.

Proof: 1. Since Lemma 2 is equivalent to the Dunford-Pettis criterion, the result follows.

2. For $p>1$, a closed bounded set is $\sigma\left(l^{p}(\pi), l^{q}(\pi)\right)$-compact. But it is also $\sigma\left(l^{1}(\pi), l^{\infty}(\pi)\right)$-compact, since $l^{p}(\pi) \subset l^{1}(\pi)$ and $l^{\infty}(\pi) \subset l^{q}(\pi)$. Apply statement 1 .

Claim 4 Assume AO. If $b^{i}=+\infty$ for all $i$, then the allocation set $\mathcal{A}$ is $l^{1}(\pi)$ compact. The set $\mathcal{U}$ is also compact.

Proof: The idea of the proof is that, if the attainable allocation sequence does not belong to a weakly compact set, then for some state $s$, there will be an agent $i$ such that $x_{s}^{i}$ tends to $+\infty$ and an agent $j$ such that $x_{s}^{j}$ tends to $-\infty$. Then by reducing $x_{s}^{i}$ and increasing $x_{s}^{j}$, the value of $U^{i}\left(x^{i}\right)$ does not diminish 
very much. Because $b^{j}=+\infty$, the value of $U^{j}\left(x^{j}\right)$ will become very large, even tends to infinity, and that leads us to a contradiction with the bounded property of $\mathcal{U}$.

Assume the contrary: $\mathcal{A}$ is not compact. Then from Claim 2, there exists a sequence $\left\{\left(x^{1}(n), x^{2}(n), \ldots, x^{m}(n)\right)\right\}_{n} \subset \mathcal{A}$, an agent $i$ and a constant $\epsilon>0$ such that

$$
\forall n, \sum_{s=n}^{\infty} \pi_{s}^{i}\left|x_{s}^{i}(n)\right|>\epsilon
$$

Denote for all $k, v^{k}:=\limsup _{n \rightarrow \infty} U^{k}\left(x^{k}(n)\right)$.

By Proposition $1, \mathcal{A}$ is bounded in $l^{1}(\pi)$. We can suppose, without loss generality, that $\sum_{s=n}^{\infty} \pi_{s}^{i}\left|x_{s}^{i}(n)\right| \rightarrow c^{i}>0$ when $n \rightarrow \infty$. This implies $\lim _{n} \sum_{s=n}^{\infty} \pi_{s}^{i} x_{s}^{i+}(n)-$ $\lim _{n} \sum_{s=n}^{\infty} \pi_{s}^{i} x_{s}^{i-}(n)=c^{i}$. The limits of these two sums exist because $x^{i} \in l^{1}(\pi)$. We know that $\sum_{j \neq i} x_{s}^{i}(n)=\bar{e}_{s}-x_{s}^{i}(n)$. So, for every $s, \exists j$ such that $x_{s}^{j}(n) \leq$ $-\frac{x_{s}^{i}(n)-\left|\bar{e}_{s}\right|}{m-1}$. Since there is a finite number of agents $j \neq i$, we can assume that, for simplicity, there exist $i$ and $j$ which satisfy two properties:

(i) $\exists E_{n}^{i} \subset \mathbb{N} \cap\{s \geq n\}, x_{s}^{i}>0$ for all $s \in E_{n}^{i}$ and

$$
\lim _{n} \sum_{s \in E_{n}^{i}} \pi_{s}^{i} x_{s}^{i}(n)=c^{i}>0 .
$$

(ii) For all $s \in E_{n}^{i}$

$$
x_{s}^{j}(n) \leq-\frac{x_{s}^{i}(n)-\left|\bar{e}_{s}\right|}{m-1} .
$$

With each $M>0$, define the set $S_{n}^{i} \subset E_{n}^{i}$ as follows

$$
S_{n}^{i}=\left\{s: x_{s}^{i}(n)>\left|\bar{e}_{s}\right|+M(m-1)\right\} .
$$

We have an observation: $\lim _{n} \sum_{E_{n}^{i} \backslash S_{n}^{i}} \pi_{s}^{i} x_{s}^{i}(n)=0$. Indeed

$$
\begin{aligned}
\lim _{n \rightarrow \infty} \sum_{s \in E_{n}^{i} \backslash S_{n}^{i}} \pi_{s}^{i} x_{s}^{i}(n) & \leq \sum_{s \in E_{n}^{i} \backslash S_{n}^{i}} \pi_{s}^{i}\left(\left|\bar{e}_{s}\right|+M(m-1)\right) \\
& \leq \sum_{s=n}^{\infty} \pi_{s}^{i}\left|\bar{e}_{s}\right|+M(m-1) \sum_{s=n}^{\infty} \pi_{s}^{i}
\end{aligned}
$$

which tends to zero, since $\bar{e} \in l^{1}(\pi)$.

Hence we have $S_{n}^{i} \neq \emptyset$ for all $n$ big enough, and

$$
\lim _{n \rightarrow \infty} \sum_{s \in S_{n}^{i}} \pi_{s}^{i} x_{s}^{i}(n)=c^{i}
$$

We have

$$
x_{s}^{j}(n) \leq \frac{\left|\bar{e}_{s}\right|-x_{s}^{i}(n)}{m-1}<-M
$$


Since $\pi^{i}$ and $\pi^{j}$ are equivalent, we can assume that $\lim _{n} \sum_{s \in S_{n}^{i}} \pi_{s}^{j} x_{s}^{i}(n)=c^{j}>$ 0 . Notice that these limits do not depend on $M$.

Define $\alpha:=\min \left(v^{k}, v^{i}-\frac{u^{i}(0) c^{i}}{m-1}\right)-1,(k=1, \ldots, m)$. Define $\mathcal{A}_{\alpha}$ the set of $\left(x^{k}\right) \in l^{1}(\pi)$ satisfies $U^{k}\left(x^{k}\right) \geq \alpha \forall k$ and $\sum x^{k}=\bar{e}$. Using the same proof as in Proposition 1, we show there exists $C>0$ such that $U^{j}\left(x^{j}\right)<C$ for all $\left(x^{1}, \ldots, x^{m}\right) \in \mathcal{A}_{\alpha}$. Notice that our sequence $\left(x^{k}(n)\right)$ belongs to $\mathcal{A}_{\alpha}$ for $n$ large enough.

Since $b^{j}=+\infty$ we can choose $M$ very big such that

$$
v^{j}+\frac{u^{j \prime}(-M) c^{j}}{m-1}>C
$$

Now consider the sequence $\left(y^{1}(n), y^{2}(n), \ldots, y^{m}(n)\right)$ defined as follows

$$
\begin{aligned}
& y_{s}^{i}(n):=x_{s}^{i}(n)-\frac{x_{s}^{i}-\left|\bar{e}_{s}\right|}{m-1}+M \text { with } s \in S_{n}^{i}, \\
& y_{s}^{j}(n):=x_{s}^{j}(n)+\frac{x_{s}^{i}-\left|\bar{e}_{s}\right|}{m-1}-M \text { with } s \in S_{n}^{i} .
\end{aligned}
$$

Let $y_{s}^{k}=x_{s}^{k}$ with every $k \neq i, j$ or $s \notin S_{n}^{i}$.

Notice that $\sum_{i} y^{i}(n)=\bar{e}$, and $y_{s}^{i}(n) \leq x_{s}^{i}(n), y_{s}^{j}(n) \geq x_{s}^{j}(n)$ for all $s$. We will prove that $\left\{U^{l}\left(y^{l}(n)\right)\right\}_{l=1, m}$ is bounded below by $\alpha$, but $U^{j}\left(y^{j}(n)\right)$ is not bounded above by $C$. And this is a contradiction.

Indeed,

$$
\begin{aligned}
U^{i}\left(y^{i}(n)\right)-U^{i}\left(x^{i}(n)\right) & =\sum_{s \in S_{n}^{i}} \pi_{s}^{i}\left(u^{i}\left(y_{s}^{i}(n)\right)-u^{i}\left(x_{s}^{i}(n)\right)\right) \\
& \geq \sum_{s \in S_{n}^{i}} \pi_{s}^{i} u^{i \prime}\left(x_{s}^{i}(n)-\frac{x_{s}^{i}(n)-\left|\bar{e}_{s}\right|}{m-1}+M\right)\left(-\frac{x_{s}^{i}(n)-\left|\bar{e}_{s}\right|}{m-1}+M\right) \\
& \geq \sum_{s \in S_{n}^{i}} \pi_{s}^{i} u^{i \prime}(M)\left(-\frac{x_{s}^{i}(n)}{m-1}\right)+u^{i \prime}(M)\left(\frac{\left|\bar{e}_{s}\right|}{m-1}+M\right) \sum_{s \in S_{n}^{i}} \pi_{s}^{i} \\
& \geq-\frac{u^{i \prime}(M)}{m-1} \sum_{s \in S_{n}^{i}} \pi_{s}^{i} x_{s}^{i}(n)+u^{i \prime}(M)\left(\frac{\left|\bar{e}_{s}\right|}{m-1}+M\right) \sum_{s \in S_{n}^{i}} \pi_{s}^{i} .
\end{aligned}
$$

When $n \rightarrow \infty$, the second term of the right hand side term in the inequality above tends to zero while first term tends to $-\frac{u^{i \prime}(M) c^{i}}{m-1}$. Thus,

$$
\limsup _{n \rightarrow \infty} U^{i}\left(y^{i}(n)\right) \geq v^{i}-\frac{u^{i \prime}(M) c^{i}}{m-1} \geq v^{i}-\frac{u^{i \prime}(0) c^{i}}{m-1}>\alpha .
$$

For $n$ large enough, $U^{k}\left(y^{k}(n)\right)$ is bounded below by $\alpha, \forall k \neq j$. Then we can 
estimate the limit of $U^{j}\left(y^{j}(n)\right)$ when $n \rightarrow \infty$,

$$
\begin{aligned}
U^{j}\left(y^{j}(n)\right)-U^{j}\left(x^{j}(n)\right) & =\sum_{s \in S_{n}^{i}} \pi_{s}^{j}\left(u^{j}\left(y_{s}^{j}(n)\right)-u^{j}\left(x_{s}^{j}(n)\right)\right) \\
& \geq \sum_{s \in S_{n}^{i}} \pi_{s}^{j} u^{j^{\prime}}\left(x_{s}^{j}(n)+\frac{x_{s}^{i}(n)-\left|\bar{e}_{s}\right|}{m-1}-M\right)\left(\frac{x_{s}^{i}(n)-\left|\bar{e}_{s}\right|}{m-1}-M\right) \\
& \geq \sum_{s \in S_{n}^{i}} \pi_{s}^{j} u^{j^{\prime}}(-M)\left(\frac{x_{s}^{i}(n)-\left|\bar{e}_{s}\right|}{m-1}-M\right) \\
& \geq \frac{u^{j^{\prime}}(-M)}{m-1} \sum_{s \in S_{n}^{i}} \pi_{s}^{j} x_{s}^{i}(n)-M u^{j^{\prime}}(-M) \sum_{s \in S_{n}^{i}} \pi_{s}^{j}-\frac{u^{j^{\prime}}(-M)}{m-1} \sum_{s \in S_{n}^{i}}\left|\bar{e}_{s}\right| \pi_{s}^{j} .
\end{aligned}
$$

Take the limit

$$
\limsup _{n \rightarrow \infty} U^{j}\left(y^{j}(n)\right) \geq v^{j}+\frac{u^{j^{\prime}}(-M) c^{j}}{m-1}>C .
$$

A contradiction. Hence $\mathcal{A}$ is $l^{1}(\pi)$-compact.

The proof of Claim 6 requires Claim 5

Claim 5 Suppose that $\mathcal{A}$ is bounded and $\left(v^{1}, v^{2}, \ldots, v^{m}\right)$ is in the closure of $\mathcal{U}$. Suppose that there exists a sequence $\{x(n)\}_{n} \subset \mathcal{A}$ such that there exists $i$ such that $\lim _{n} U^{i}\left(x^{i}(n)\right)>v^{i}$, and for all $j \neq i, \lim _{n} U^{j}\left(x^{j}(n)\right) \geq v^{j}$. Then $\left(v^{1}, v^{2}, \ldots, v^{m}\right) \in \mathcal{U}$.

Proof: Fix $t \in \mathbb{N}$ arbitrarily. Let $C>0$ be the upper bound of $\mathcal{A}$ in $l^{1}(\pi)$, we know that $\left|x_{t}^{j}(n)\right|<\frac{C}{\pi_{t}^{j}}$ for all $j$ and all $n$. Fix some $j$. We define the sequence $\left\{y^{k}(n)\right\}_{k=1, \ldots, m}$ as follows

$$
\begin{aligned}
y^{k}(n) & =x^{k}(n) \text { if } k \neq i, j, \\
y_{s}^{i}(n) & =x_{s}^{i}(n) \text { if } s \neq t, \\
y_{t}^{i}(n) & =x_{t}^{i}(n)-\epsilon, \\
y_{t}^{j}(n) & =x_{t}^{j}(n)+\epsilon .
\end{aligned}
$$

For $k \neq i, j, \lim _{n} U^{k}\left(y^{k}(n)\right)=v^{k}$. And we have

$$
\begin{aligned}
U^{i}\left(y^{i}(n)\right)-U^{i}\left(x^{i}(n)\right) & =\pi_{t}^{i}\left(u^{i}\left(y_{t}^{i}(n)\right)-u^{i}\left(x_{t}^{i}(n)\right)\right) \\
& \geq \pi_{t}^{i}(-\epsilon) u^{i \prime}\left(x_{t}^{i}(n)-\epsilon\right) \geq-\epsilon \pi_{t}^{i} u^{i \prime}\left(-\frac{C}{\pi_{t}^{i}}-\epsilon\right)
\end{aligned}
$$

and

$$
\begin{aligned}
U^{j}\left(y^{j}(n)\right)-U^{j}\left(x^{j}(n)\right) & =\pi_{t}^{j}\left(u^{j}\left(y_{t}^{j}(n)\right)-u^{j}\left(x_{t}^{j}(n)\right)\right) \\
& \geq \pi_{t}^{j} \epsilon u^{j \prime}\left(x_{t}^{j}(n)+\epsilon\right) \geq \epsilon \pi_{t}^{j} u^{j \prime}\left(\frac{C}{\pi_{t}^{j}}+\epsilon\right) .
\end{aligned}
$$


Since $\liminf { }_{n} U^{i}\left(x^{i}(n)\right)>v^{i}$, by choosing $\epsilon$ small enough, the sequence $\{y(n)\}_{n}$ will satisfy $\liminf _{n} U^{i}\left(y^{i}(n)\right)>v^{i}$ and $\liminf _{n} U^{j}\left(y^{j}(n)\right)>v^{j}$.

By induction we can find a sequence $\left\{z^{k}(n)\right\}_{n} \subset \mathcal{A}$ which satisfies $\lim _{n} U^{k}\left(z^{k}(n)\right)>$ $v^{k}$ for all $k=1,2, \ldots, m$. Hence $\left(v^{1}, v^{2}, \ldots, v^{m}\right) \in \mathcal{U}$.

Claim 6 Assume AO. If $a^{i}=0$ for all $i$, then $\mathcal{U}$ is compact.

Proof: Since the (NA) condition holds, from Proposition 1, we know that $\mathcal{U}$ is bounded. We will prove that $\mathcal{U}$ is closed. Suppose that $\left(v^{1}, \ldots, v^{m}\right)$ belong to the closure of $\mathcal{U}$ and the sequence $\{x(n)\}_{n} \subset \mathcal{A}$ such that $\lim _{n} U^{i}\left(x^{i}(n)\right)=v^{i}$.

If the sequence $\{x(n)\}_{n}$ belongs to a compact set of $l^{1}(\pi)$, without loss of generality, we can suppose that $\lim _{n} x^{i}(n)=x^{i}$ in this topology. Since $U^{i}$ is continuous, we have $U^{i}\left(x^{i}\right) \geq v^{i}$ for all $i$. Thus $\left(v^{1}, \ldots, v^{m}\right) \in \mathcal{U}$.

If the sequence $\{x(n)\}_{n}$ does not belong to a compact set, we can suppose that there exists $c>0$ such that for an agent $i$

$$
\lim _{n \rightarrow \infty} \sum_{s=n}^{\infty} \pi_{s}^{i}\left|x_{s}^{i}(n)\right|=c .
$$

As in the proof of Claim 4, we can choose a pair $(i, j)$ which satisfies the two properties:

(i) $\exists E_{n}^{i} \subset \mathbb{N} \cap\{s \geq n\}, x_{s}^{i}>0$ for all $s \in E_{n}^{i}$ and

$$
\lim _{n} \sum_{s \in E_{n}^{i}} \pi_{s}^{i} x_{s}^{i}(n)=c^{i}>0 .
$$

(ii) For all $s \in E_{n}^{i}$

$$
x_{s}^{j}(n) \leq-\frac{x_{s}^{i}(n)-\left|\bar{e}_{s}\right|}{m-1} .
$$

With each $M>0$, define the set $S_{n}^{i} \subset E_{n}^{i}$ as follows

$$
S_{n}^{i}=\left\{s: x_{s}^{i}(n)>\left|\bar{e}_{s}\right|+M(m-1)\right\} .
$$

We have an observation: $\lim _{n} \sum_{E_{n}^{i} \backslash S_{n}^{i}} \pi_{s}^{i} x_{s}^{i}(n)=0$. Indeed

$$
\begin{aligned}
\lim _{n \rightarrow \infty} \sum_{s \in E_{n}^{i} \backslash S_{n}^{i}} \pi_{s}^{i} x_{s}^{i}(n) & \leq \sum_{s \in E_{n}^{i} \backslash S_{n}^{i}} \pi_{s}^{i}\left(\left|\bar{e}_{s}\right|+M(m-1)\right) \\
& \leq \sum_{s=n}^{\infty} \pi_{s}^{i}\left|\bar{e}_{s}\right|+M(m-1) \sum_{s=n}^{\infty} \pi_{s}^{i}
\end{aligned}
$$

which tends to zero, since $\bar{e} \in l^{1}(\pi)$.

Hence we have $S_{n}^{i} \neq \emptyset$ for all $n$ big enough, and

$$
\lim _{n \rightarrow \infty} \sum_{s \in S_{n}^{i}} \pi_{s}^{i} x_{s}^{i}(n)=c^{i}
$$


We have

$$
x_{s}^{j}(n) \leq \frac{\left|\bar{e}_{s}\right|-x_{s}^{i}(n)}{m-1}<-M .
$$

Since $\pi^{i}$ and $\pi^{j}$ are equivalent, we can assume that $\lim _{n} \sum_{s \in S_{n}^{i}} \pi_{s}^{j} x_{s}^{i}(n)=c^{j}>$ 0 . Notice that these limits do not depend on $M$.

Define $\alpha:=\min \left(v^{k}, v^{i}-\frac{u^{i \prime}(0) c^{i}}{m-1}\right)-1,(k=1, \ldots, m)$. Define $\mathcal{A}_{\alpha}$ the set of $\left(x^{k}\right) \in l^{1}(\pi)$ satisfies $U^{k}\left(x^{k}\right) \geq \alpha \forall k$ and $\sum x^{k}=\bar{e}$. From Proposition 1 we know that there exists $C>0$ such that $U^{j}\left(x^{j}\right)<C$ for all $\left(x^{1}, \ldots, x^{m}\right) \in \mathcal{A}_{\alpha}$. Notice that our sequence $\left(x^{k}(n)\right) \in \mathcal{A}_{\alpha}$ for $n$ large enough. Fix $\epsilon>0$. Since $u^{i \prime}(+\infty)=0$ we can choose $M>0$ such that $u^{i \prime}(M)<(m-1) \epsilon / c$. By similar arguments as in the proof of Claim 4 , we can construct the sequence $\left(y^{k}(n)\right)$ such that:

$$
\begin{aligned}
& \lim _{n \rightarrow \infty} U^{i}\left(y^{i}(n)\right) \geq v^{i}-\frac{u^{i \prime}(M) c^{i}}{m-1} \\
& \lim _{n \rightarrow \infty} U^{j}\left(y^{j}(n)\right) \geq v^{j}+\frac{u^{j \prime}(-M) c^{j}}{m-1} \\
& \lim _{n \rightarrow \infty} U^{k}\left(y^{k}(n)\right)=v^{k} \text { for all } k \neq i, j
\end{aligned}
$$

with $c^{i}, c^{j}>0$ and $c^{i}<c$ and $c^{i}$ and $c^{j}$ do not depend on $M$.

So, for $n$ large enough, $U^{i}\left(y^{i}(n)\right)>v^{i}-\epsilon$, and for all $k \neq i, j, U^{k}\left(y^{k}(n)\right)>$ $v^{k}-\epsilon$ whereas $\lim _{n} U^{j}\left(y^{j}(n)\right)=v^{j}+\frac{u^{j^{\prime}}(-M) c^{j}}{m-1}>v^{j}+\frac{u^{j^{\prime}}(0) c^{j}}{m-1}>v^{j}$. Let $\epsilon \rightarrow 0$ and by applying Claim 5 , we have $\left(v^{1}, v^{2}, \ldots, v^{m}\right) \in \mathcal{U}$.

\section{Proof of Theorem 1}

This is a direct consequence of Claims 4, 6 and 1.

The proof of the last theorem, Theorem 2, requires Claims 7 and 8 .

Claim 7 Assume AO. Assume that there is only one risk neutral agent, $I_{2}=$ $\left\{i_{0}\right\}$. If $a^{i}=0$ and $b^{i}=+\infty$ for all $i \in I_{1}$, then (NA) holds, and $\mathcal{U}$ is compact.

Proof: Condition (NA) holds as a direct consequence of Claim 1. Now we prove that $\mathcal{U}$ is compact.

Suppose that the feasible sequence $\left\{x_{n}\right\}$ satisfies $\lim _{n} U^{i}\left(x_{n}^{i}\right)=v^{i}$ for every $i$. We prove that $\left(v^{1}, \ldots, v^{m}\right) \in \mathcal{U}$.

First case: the sequence $\left\{x_{n}\right\}$ belongs to a compact set, we have $\left(v^{1}, v^{2}, \ldots, v^{m}\right)$ belongs to $\mathcal{U}$.

Second case: the sequence $\left\{x_{n}\right\}$ does not belong to a compact set. By using the same arguments in the proof of Proposition 4, there exist $i$ and $j$ which satisfy two properties: 
(i) $\exists E_{n}^{i} \subset \mathbb{N} \cap\{s \geq n\}, x_{s}^{i}>0$ for all $s \in E_{n}^{i}$ and

$$
\lim _{n} \sum_{s \in E_{n}^{i}} \pi_{s}^{i} x_{s}^{i}(n)=c^{i}>0 .
$$

(ii) For all $s \in E_{n}^{i}$

$$
x_{s}^{j}(n) \leq-\frac{x_{s}^{i}(n)-\left|\bar{e}_{s}\right|}{m-1} .
$$

If $j \neq i_{0}$, since $b^{j}=+\infty$, by using the same arguments as in the proof of Proposition 4 we have a contradiction.

This implies $j=i_{0}$. Hence $i \neq i_{0}$, and we have $a^{i}=0$. By using the same arguments in the proof of Proposition 6 , we have $\left(v^{1}, \ldots, v^{m}\right) \in \mathcal{U}$.

Hence $\mathcal{U}$ is compact.

Claim 8 Let $f^{1}, \ldots, f^{n}$, be $n$ vectors in $l^{p}(\pi)$, and $p \in l^{\infty}(\pi)$. Take any $x \in$ $l^{p}(\pi)$ such that $p \cdot x=p \cdot \sum_{i} f^{i}$. Then there exists $x^{1}, \ldots, x^{n}$ in $l^{p}(\pi)$ such that $\sum_{i} x^{i}=x$ and $p \cdot x^{i}=p \cdot f^{i}$ for all $i$.

Proof: This is true for $n=1$. Suppose that the claim is true for $n-1$. Take any $x^{1}$ such that $p \cdot x^{1}=p \cdot f^{1}$. We have $p \cdot\left(x-x^{1}\right)=p \cdot \sum_{i=2}^{n} f^{i}$. Using the hypothesis of induction, there exists $x^{2}, \ldots, x^{n}$ such that $\sum_{i=2}^{n} x^{i}=x-x^{1}$ and $p \cdot x^{i}=p \cdot f^{i}$ for all $i$.

\section{Proof of Theorem 2}

In the proof of Proposition 2, we have $\pi^{i}=\pi^{j}=\pi^{I}$ and $\lambda_{i} a^{i}=\lambda_{j} a^{j}=\zeta$, for all $i, j \in I_{2}$. For $x^{I} \in l^{1}(\pi)$ satisfying $x^{I}=\sum_{i \in I_{2}} x^{i}$, with $x^{i} \in l^{1}(\pi)$, define

$$
U^{I}\left(x^{I}\right)=\sum_{i \in I_{2}} \lambda_{i} U^{i}\left(x^{i}\right)
$$

We consider now the economy $\mathcal{E}^{I}$ with $\left|I_{1}\right|+1$ agents, $\left|I_{1}\right|$ agents who are risk averse, with endowment $e^{i}$, utility function $U^{i}$, and the last agent (denoted by $I$ ) with endowment $e^{I}=\sum_{i \in I_{2}} e^{i}$, utility function $U^{I}$. It is easy to verify that agent $I$ is risk neutral, with

$$
U^{I}\left(x_{s}^{I}\right)=\zeta \sum_{s=1}^{\infty} \pi_{s}^{I} x_{s}^{I},
$$

and the new economy satisfies (NA) condition. By Claim 7, this economy has a compact rational utility set and have an equilibrium, denote by $\left(p^{*}, x^{*}\right)$. For all $i \in I_{1}, x^{* i}$ is the solution to

$$
\begin{aligned}
& \max \sum_{s=1}^{\infty} \pi_{s}^{i} u^{i}\left(x_{s}^{i}\right) \\
& \text { s.t. } \sum_{s=1}^{\infty} p_{s}^{*} x_{s}^{i}=\sum_{s=1}^{\infty} p_{s}^{*} e_{s}^{i} .
\end{aligned}
$$


and $x^{* I}$ is the solution to

$$
\begin{aligned}
& \max U^{I}\left(x^{I}\right) \\
& \text { s.t. } \sum_{s=1}^{\infty} p_{s}^{*} x_{s}^{I}=\sum_{s=1}^{\infty} p_{s}^{*} e_{s}^{I} .
\end{aligned}
$$

If $U^{I}\left(x^{i}\right)>U^{I}\left(x^{* I}\right)$, then $p^{*} \cdot x^{i}>p^{*} \cdot e^{I}$.

By the same arguments as in the proof of Claim 1, there exists $\lambda_{i}^{*}>0$, $\lambda_{I}>0$ such that for $i \in I_{1}, p_{s}^{*}=\lambda_{i} \pi_{s}^{i} u^{i \prime}\left(x_{s}^{* i}\right)$. For $I$, we have $p_{s}^{*}=\zeta \pi_{s}^{I}=\lambda_{i} a^{i} \pi_{s}^{I}$, $\forall i \in I_{2}, \forall s$. The function $U^{I}$ is strictly increasing, so

$$
p^{*} \cdot x^{* I}=p^{*} \cdot e^{* I}=p^{*} \cdot \sum_{i \in I_{2}} e^{i} .
$$

By Claim 8, for all $i \in I_{2}$ there exists $x^{* i} \in l^{p}(\pi)$ such that $\sum_{i \in I_{2}} x^{* i}=x^{* I}$, and $p^{*} \cdot x^{* i}=p^{*} \cdot e^{i}, \forall i$.

Fix $i \in I_{2}$. Take $x^{i}$ such that $U^{i}\left(x^{i}\right)>U^{i}\left(x^{* i}\right)$. We prove that $p^{*} \cdot x^{i}>p \cdot e^{i}$. Indeed, we have

$$
U^{i}\left(x^{i}\right)=\sum_{s=1}^{\infty} a^{i} \pi_{s}^{i} x_{s}^{i}=\frac{1}{\lambda_{i}} \sum_{s=1}^{\infty} \lambda_{i} a^{i} \pi_{s}^{i}=\frac{1}{\lambda_{i}} \sum_{s=1}^{\infty} p_{s}^{*} x_{s}^{i} .
$$

Hence $U^{i}\left(x^{i}\right)>U^{i}\left(x^{* i}\right)$ implies $p^{*} \cdot x^{i}>p^{*} \cdot x^{* i}=p^{*} \cdot e^{i}$.

From Claim 1, the equilibrium price $p^{*}$ is in $l^{q}(\pi)$ with $1 \leq p<+\infty$, $\frac{1}{p}+\frac{1}{q}=1$ and if $p=+\infty$, then $p^{*} \in l^{1}(\pi)$. We have proved that $\left(p^{*},\left(x^{* i}\right)_{i}\right)$ is an equilibrium of the model.

Conversely, suppose that the model has an equilibrium, then by using Proposition 2, we have (NA). The proof of Theorem 2 is complete.

\section{References}

[1] Allouch, N., C. Le Van and F.H. Page (2002): The geometry of arbitrage and the existence of competitive equilibrium. Journal of Mathematical Economics 38, 373-391.

[2] Arrow, K. J., L. Hurwicz, and H. Uzawa (1958): Programming in Linear spaces. Stanford University Press, Stanford, California.

[3] Brown, D. and J. Werner (1995): Existence theorems in the classical asset pricing model. Econometrica 59, 1169-1174.

[4] Brown, D. and J. Werner (1995): Arbitrage and existence of equilibrium in infinite asset markets. Review of Economic Studies, 62, 101-114. 
[5] Cheng, H. (1991): Asset market equilibrium in infinite dimensional complete markets. Journal of Mathematical Economics, 20, 137-152.

[6] Chichilnisky, G. and G. Heal (1993): Competitive Equilibrium in Sobolev Spaces without Bounds on Short Sales, Journal of Economic Theory, 59, 364-384.

[7] Dana, R. A and C. Le Van (1996): Asset Equilibria in $L^{p}$ Spaces: A Duality Approach. Journal of Mathematical Economics, 25, 263-280.

[8] Dana, R. A and C. Le Van (2010): Overlapping risk adjusted sets of priors and the existence of efficient allocations and equilibria with short-selling. Journal of Economic Theory, 145, 2186-2202.

[9] Dana, R.A, C. Le Van and F. Magnien (1997): General Equilibirum in asset markets with or without short-selling. Journal of Mathematical Analysis and Applications 206, 567-588.

[10] Dana, R.A, C.Le Van and F.Magnien (1999): On the different notions of arbitrage and existence of equilibrium. Journal of Economic Theory 86, 169-193.

[11] Daher, W, Martins-da-Rocha, V.F and Vailakis, Y (2007): Asset market equilibrium with short-selling and differential information. Economic Theory 32, 425-446.

[12] Dunford, N. and J. T. Schwartz (1966), "Linear Operators, Part I: general Theory," 3rd ed., Interscience, New York.

[13] Geistdorfer-Florenzano, M. (1982): The Gale-Nikaido-Debreu Lemma and the Existence of Transitive Equilibrium With or Without the Free Disposal Assumption. Journal of Mathematical Economics 9, 113-134.

[14] Hart, O. (1974): On the Existence of an Equilibrium in a Securities Model. Journal of Economic Theory 9, 293-311.

[15] Le Van, C. (1996); Complete characterization of Yannelis-Zame and Chichilnisky-Kalman-Mas-Colell properness conditions on preferences for separable concave functions defined in $L_{+}^{p}$ and $L^{p}$. Economic Theory, 8, $155-166$.

[16] Le Van, C. and D.H. Truong Xuan (2001): Asset market equilibrium in $L^{p}$ spaces with separable utilities. Journal of Mathematical Economics 36, 241-254.

[17] Nielsen, L. T (1989): Asset market equilibrium with short-selling. Review of Economic Studies, 41, 392-469. 
[18] Page, F.H (1987): On equilibrium in Hart's securities exchange model. Journal of Economic Theory 41, 392-404.

[19] Page, F.H. Jr, and M.H. Wooders, (1996): A necessary and sufficient condition for compactness of individually rational and feasible outcomes and existence of an equilibrium. Economics Letters 52, 153-162.

[20] Page, F.H. Jr, M.H. Wooders and P.K. Monteiro (2000): Inconsequential arbitrage. Journal of Mathematical Economics 34, 439-469.

[21] Rockafellar, R.T (1970): Convex Analysis, Princeton University Press, Princetion, New-Jersey.

[22] Werner, J. (1987): Arbitrage and the Existence of Competitive Equilibrium. Econometrica 55, 1403-1418.

[23] Won, D., and N.C. Yannelis: Equilibrium Theory with Unbounded Consumption Sets and Non Ordered Preferences, Part I. Journal of Mathematical Economics 44, 1266-1283. 